\title{
Distributed Data Vending on Blockchain
}

\author{
Jiayu Zhou ${ }^{1}$, Fengyi Tang ${ }^{1}$, He Zhu ${ }^{2}$, Ning $\mathrm{Nan}^{2}$, Ziheng Zhou ${ }^{3}$ \\ ${ }^{1}$ Computer Science and Engineering, Michigan State University, East Lansing, MI 48824, USA \\ ${ }^{2}$ BitOcean Ltd., Tokyo, Japan \\ ${ }^{3}$ Vechain Foundation, Singapore \\ Email: \{jiayuz, tangfeng\}@msu.edu, \{hezhu, nanxiaoning\}@bitocean.com, peter.zhou@vechain.com
}

\begin{abstract}
Recent advances in blockchain technologies have provided exciting opportunities for decentralized applications. Specifically, blockchain-based smart contracts enable credible transactions without authorized third parties. The attractive properties of smart contracts facilitate distributed data vending, allowing for proprietary data to be securely exchanged on a blockchain. Distributed data vending can transform domains such as healthcare by encouraging data distribution from owners and enabling large-scale data aggregation. However, one key challenge in distributed data vending is the trade-off dilemma between the effectiveness of data retrieval, and the leakage risk from indexing the data. In this paper, we propose a framework for distributed data vending through a combination of data embedding and similarity learning. We illustrate our framework through a practical scenario of distributing and aggregating electronic medical records on a blockchain. Extensive empirical results demonstrate the effectiveness of our framework.

Index Terms - blockchain, big data, machine learning, healthcare, electronic medical records
\end{abstract}

\section{INTRODUCTION}

Recent years have witnessed surging interests in blockchain technology [27], an open and decentralized ledger that links transaction records using blocks and provides proven security backed by cryptography. Blockchain was first introduced as the infrastructure supporting the electronic cash system Bitcoin [20], and is now transforming a wide domain of industries such as supply chains [28], biomedical research [14], healthcare [6], [13], [23], financial transactions [9], networking [37] and social networks [9]. Of particular interest is the Ethereum blockchain [31], which has enabled credible transactions without trusted third parties by introducing smart contracts, with small programs running on top of the blockchain to provide transparent and secured contracting with reduced transaction costs.

The advent of blockchain infrastructures paved the way for a new domain of data vending, allowing individual data owners to directly benefit from sharing proprietary data over the blockchain. In the era of big data, vast amount of data collected has been widely used to improve decision making for industries, namely through building personalized recommender systems [12], [15] and targeted advertisement [5]. As a result, organizations that collect and aggregate data at scale stand to profit enormously in the process. However, as data stakeholders, the users from whom the data is collected from rarely get their share of dividends despite significant contributions to the fortune. In fact, in most cases, organizations regard collected data as their private assets and prevent the data

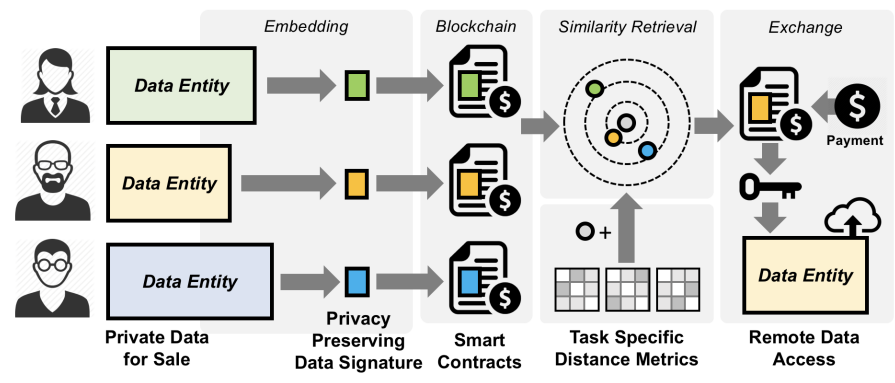

Fig. 1: Illustration of the proposed distributed data vending (DDV) framework. DDV enables private data to be exchanged through smart contracts.

from being shared even for research purposes, which could otherwise contribute to the advancement of our society.

Perhaps a prime example lies in our healthcare system, where electronic health records (EHR) systems are now deployed in most hospitals across the United States. In recent years, medical histories in EHR have been used to build data-driven models to improve healthcare resource management [35], [36]. Analyses of the EHR data often reveal important insights into the underlying pathophysiology of numerous complicated diseases [34]. These discoveries are invaluable to the development of drugs and treatments. However, the patients who own the data [7] and contribute to research outcomes are rarely rewarded for their contributions, despite the hiking healthcare costs which accompany the rise of healthcare information exchange in recent years [8], [16]. On the other hand, many research institutions such as universities have an extremely difficult time to access existing health data because of strict regulations such as HIPPA [21] and bureaucracy, even though there are individuals willing to share their medical histories, especially when they are incentivized to do so.

In this paper, we propose a distributed data vending ( $D D V)$ framework which consists of a suite of machine learning modules to enable individuals to exchange data on blockchains through smart contracts. Like other vending services, a data entry has to be indexed and retrieved before it can be exchanged on blockchains. However, the challenge comes from the dilemma between data indexing and information leakage: building indexes for retrieval requires access to the data, which is hard to achieve on blockchains where no trusted third parties are available. To tackle this challenge, the proposed DDV approach generates a signature of the data entry using a 
privacy-preserving data embedding procedure. The signature can be published along with the data access in smart contracts. The framework also provides task-specific similarity functions, which take the signatures as input to measure the similarity among data entries. The proposed DDV framework can be used in many data vending scenarios, and in this paper we use EHR data vending as a concrete example to illustrate the concept and its feasibility. We also provide extensive empirical evaluations to demonstrate the effectiveness of the proposed framework.

\section{RELATED WORK}

Even though the distributed data vending is a rather new problem, the challenges of this problem are closely related to many existing studies, such as health data management based on blockchain, data embedding techniques and distance metric learning in machine learning. We will briefly survey these areas and point out the relationship to this study.

\section{A. Blockchain Applications on Healthcare Data}

The properties of blockchain make it a promising tool in many health informatics applications [13]: from building decentralized backbones for health data exchange and interoperability, protocols enforced by immutable ledgers that keep track of clinical research [26], data provenance and robustness of medicine production through pharmaceutical supply chain [2], [25], to maintain patient privacy and EHR security through the persistence of consent statements in blockchain [3]. The proposed DDV framework utilizes an existing blockchain infrastructure to facilitate data exchange, and provides an effective tool to collect medical data and thus accelerate medical research.

\section{B. Medical Information Exchange on Blockchain}

Of particular interests is the domain of medical information exchange, where many studies have been done to facilitate the second use of EHR data for clinical/biomedical research. Examples include MedRec [1], [6], Healthbank [18] and ModelChain [14]. MedRec [1], [6] is closely related to the proposed research. It proposed a blockchain-based data management system to consolidate the fragmented medical records. The system involves patients in the data life cycle, allowing the patients to take control of the permission of their own medical records, and encouraging medical stakeholders to participating the blockchain network with incentives of aggregated data. The work delivered an important message that patients are one of the data owners, can serve an important role in data sharing among stakeholders, and should be controlling the data sharing process. However, one major issue in this framework is that the data is persistent in a data center of the hospital system, which requires the API exposure for remote access. Such practice is usually hard to implement due to the excessive security risks of external APIs. Moreover, since query strings of the database are persistent in smart contract, it would incur significant maintenance costs during data migration [29]. In the proposed DDV framework, we make no assumptions on the physical location of data. Once the smart contracts including desired information are retrieved, the contracts can grant access to data stored anywhere in the cloud.

\section{Data Embedding and Distance Metric Learning}

In machine learning research, data embedding has been studied for decades. The embedding can be learned in a supervised fashion (e.g., linear discriminant analysis [19]) which learns a low-rank subspace that captures most of the discriminative information, or an unsupervised one (e.g., principal component analysis [10]), which learns a subspace to capture the geometric manifold of the data matrix. More recently, the advances of deep learning lead to highly nonlinear embedding techniques such as autoencoders [30] as well as recurrent neural networks for embedding time sequence data [24]. In many information retrieval systems we need to provide a distance metric to evaluate the similarity between pairs of data points, and distance metrics vary from task to task. The distance metric learning [32] is one type of supervised learning that learns a metric from data for one specific retrieval task. In medical informatics, for example, the study of patient similarity attracts much research efforts, which facilitates effective patient stratification and discovers important risk factors [4].

In this paper, we propose to use a two-stage approach to combine the data embedding and distance metric learning, which firstly learns a privacy-preserving signature to effectively summarize the original private data, which is then used to compute similarity by a chosen similarity metric learned from database, to achieve retrieval of smart contracts, without compromising the integrity of the private data.

\section{Privacy-Preserving Data Vending}

Data vending is the exchange of private data between individual data providers (owners) and data consumers. The purpose of distributed data vending (DDV) is to enable data providers to use existing blockchains as infrastructure to list the data. Data consumers then retrieve data from blockchain and complete the purchase. The entire data exchange process is done without trusted third parties involved. In this section, we introduce the DDV framework and its key components. We use vending of medical records as a running example in the remaining of this paper and we note that the same technique can be extended to the setting of general data vending 1

\section{A. Distributed Data Vending}

Assume a data provider ( $i$ ) would like to sell some private data to others for profits and the data entity can be represented in the form of a multi-dimensional tensor $\mathcal{X}_{i} \in \mathbb{R}^{d_{1} \times \cdots \times d_{k}}$ (which subsumes the matrix case $X_{i}$ and the vector case $\mathbf{x}_{i}$ ), where $d_{1} \ldots d_{k}$ are the dimensionality of $k$ modes. For example, the purchasing history of a person can be represented in a tensor capturing [item $\times$ time $\times$ location], or the watching

\footnotetext{
${ }^{1} \mathrm{By}$ general data vending we mean the data is structured and can be represented in a vector/matrix/tensor form
} 
history for a set of users using [item $\times$ user $\times$ time] tensor [11]. And the historical medical records of a patient can be represented in the form of a sparse matrix [36] capturing [time $\times$ diagnosis $]$.

In order for potential data consumers to find the availability of $\mathcal{X}_{i}$, the data provider needs to list the information of the data to a listing service, such that the consumers can query the listing by sending a relevance criteria. The payment and data exchange can be done once the data entity is identified as relevant and fits the budget of the consumer.

Even though we are using many of such listings everyday, for example Amazon for general merchandise and Netflix for movies, a listing for general data vending is very different. Merchandise can be listed according to categories and properties, where as a movie or a song can be retrieved from its artist, genre and the year of release. However, there is no predefined categorical information for an arbitrary data tensor $\mathcal{X}_{i}$, and thus it is challenging to retrieve the data tensor needed. When vending medical data, one potential data consumer may be interested in knowing answers to query questions such as "did this patient have any diagnosis of hypertension before?" or "how many past encounters of renal failures in the past three years?", before deciding whether or not to purchase the data. In order to provide response to such query, the listing service has to know all the details of the data, i.e., the listing service has access to the entire data to be exchanged.

When the listing service is a trusted 3rd party, such query is feasible. However, such listing service is associated to an extremely high security risk: once the 3rd party listing server is compromised, all the data entries on the listing server will be leaked. Different from other listing services, once the data themselves are leaked there is no way to control the perimeter of damage. As such, there is a strong demand of a distributed data vending framework, facilitating a decentralized vending procedure that does not involve the listing service from a trusted 3rd party. Smart contracts has provided a secured and efficient tool for vending contracts on top of blockchains. However, without the 3rd party listing, the retrieval problem now involves a dilemma: On one hand, we want to enable effective retrieval for data consumers to identify the data entities of interest; on the other hand, indexing data entities requires detailed information of the data, which can leak the content of data entities before they are purchased for use.

To illustrate this leakage in the context of medical records, assume that we expose the number of diagnoses of Congestive Heart Failure (CHF) and Chronic Kidney Disease (CKD) in the smart contract to enable indexing. Any data consumer now can simply iterate through all available smart contracts in the blockchain, and the consumer can explicitly compute the marginal distributions $\operatorname{Pr}(\mathrm{CKD})$ and $\operatorname{Pr}(\mathrm{CHF})$, the joint distribution $\operatorname{Pr}(\mathrm{CKD}, \mathrm{CHF})$, as well as conditional distribution of $\operatorname{Pr}(\mathrm{CKD} \mid \mathrm{CHF})$ and $\operatorname{Pr}(\mathrm{CKD} \mid \mathrm{CHF})$. The data leakage does not stop at statistics level, as such distributions allow one to directly build predictive models, say, using Naïve Bayes, to infer posterior probabilities of any diseases used for indexing. However, to acknowledge the data contribution to such predic-

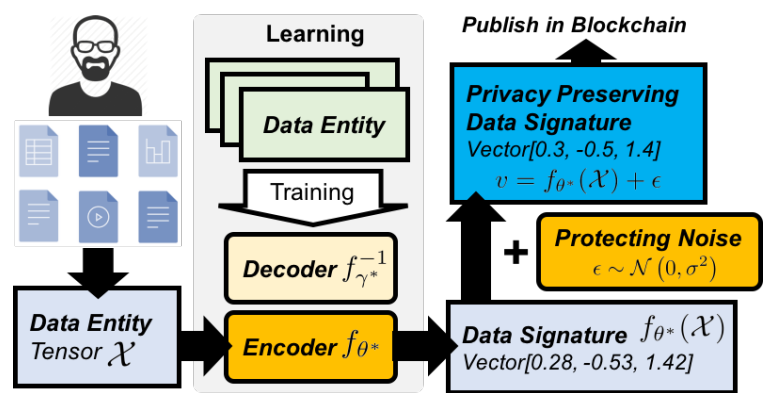

Fig. 2: Illustration of the learning of privacy-preserving signatures in DDV.

tive model, any data provided involved should be paid through executing the associated smart contracts.

In order to balance retrieval efficiency and data leakage without a 3rd party listing, we propose a distributed data vending (DDV) framework. In this framework we first embed the data into a private preserving signature vector, and then maintain a set of public similarity metrics. The signature of a data entity are publicly available in smart contracts selling the entity, and the retrieval is done by combining the data signatures and similarity metrics.

\section{B. Data Signature}

We propose to use data embedding techniques to compute data signature. The goal of data embedding is to project a data entry to a usually lower-dimensional subspace such that the data entry can be represented by a low-dimensional vector. Formally, we denote an embedding function $f$ that projects a data tensor $\mathcal{X}_{i} \in \mathbb{R}^{d_{1} \times \cdots \times d_{k}}$ to a vector $z_{i} \in \mathbb{R}^{p}$, and thus $f_{\theta}\left(\mathcal{X}_{i}\right) \rightarrow z_{i}$, where the vector $\theta$ includes a set of parameters of the projection function. One way to obtain the vector representation to extract a set of summary statistics from the tensor, and however, it is hard to warrant the effectiveness of retrieval because of the difficulty from estimating how much information are covered by summary statistics. On the other hand, different data entities may have different shapes/dimensionality, and it is challenging to design such statistics manually.

When there are many data entities available, the data embedding can be done in a data-driven way, i.e., we learn a projection function such that the subspace learned can be maximally recover the data entities. Let $\mathcal{D}=\left\{\mathcal{X}_{1}, \mathcal{X}_{2} \ldots, \mathcal{X}_{n}\right\}$ be a set of $n$ data entities available for training, the data-driven embedding is given by the following objective:

$$
\min _{\theta, \gamma} \sum_{i=1}^{n} \ell\left(\mathcal{X}_{i}, f_{\gamma}^{-1}\left(f_{\theta}\left(\mathcal{X}_{i}\right)\right)\right)
$$

where $\ell(\cdot, \cdot)$ is a loss function that evaluate the recovery error, and $f_{\gamma}^{-1}$ is an inversion function of $f$ that tries to reconstruct the data entity from the embedded vector, and $\gamma$ is the parameter of the inversion function. $f$ is usually called encoder and $f^{-1}$ is corresponding decoder.

Let $\theta^{*}, \gamma^{*}$ be an optimal solution pair to (1), then we can publish $\theta^{*}$ and the functional form of $f$, for example to a blockchain. Then any data provider who wants to sell data $\mathcal{X}_{j}$ 
can now compute the embedding $v_{j}=f_{\theta^{*}}\left(\mathcal{X}_{j}\right)$ and publish $v_{j}$ along with the smart contract. We call this embedding vector the signature of the data matrix $\mathcal{X}_{j}$. Details of this publishing will be illustrated in Sec. III-D Next we discuss two important issues associated to the embedding process.

a) Incremental Signature Learning: A high performance projection $f_{\theta}$ guarantees that the signature includes a comprehensive description of the data entry been projected. Therefore learning a good projection function is the key for effective retrieval. Since the projection function depends on the data entries $\mathcal{D}$ used for training, and usually we do not have a large amount of data to start. As more data entities are available, the projection function can be updated to improve its performance. This suggests an online learning approach to incremental update parameters of the embedding function $\theta$. For example, using:

$$
\left[\theta^{+}, \gamma^{+}\right]=\left[\theta^{-}, \gamma^{-}\right]-\tau \nabla_{[\theta, \gamma]} \ell\left(\mathcal{X}_{i}, f_{\gamma^{-}}^{-1}\left(f_{\theta^{-}}\left(\mathcal{X}_{i}\right)\right)\right),
$$

where $\tau$ is the step size for incremental update, which is converging to 0 , and the superscripts + and - denote before and after the update respectively.

b) Privacy-Preserving Signature: The rationale behind releasing the signature $v_{j}$ to public is the belief that $v_{j}$ cannot be used to recover the original data entry $X_{j}$. Conceptually this is highly likely because that the original data $X_{j}$ is in a much larger space than the signature $v_{j}$, and such projections are supposed to single directional, and given $v_{j}$ the chance of recovering the original space is small. However, because that the embedding projection is learned in a way that seeks an structured manifold of the original data in $\mathcal{D}$, it is possible to recover the original data once the shape of the manifold is obtained. And manifold information is captured in the inverse function $f_{\gamma}^{-1}$, which is why the reconstruction $f_{\gamma^{*}}^{-1}\left(f_{\theta^{*}}\left(\mathcal{X}_{i}\right)\right)$ can be quite small in practice. Therefore protecting $f_{\gamma^{*}}^{-1}$ is essential to protect the signatures. Once an accurate approximation of $f_{\gamma^{*}}^{-1}$ is obtained by adversarial parties, they can use it to recover a large amount of information by going through the signatures alone.

On the other hand, since $f$ is publicly available, it is reasonable to assume that the functional form of $f^{-1}$ is also publicly available. This implies that once the parameters in $\gamma$ are estimated, then the signatures are compromised. In fact, when someone already purchased quite a few data entries, the purchased data entries can be used to construct a dataset $\tilde{\mathcal{D}}$, which can then be used to estimate the decoder:

$$
\tilde{\gamma}=\operatorname{argmin}_{\gamma} \sum_{\mathcal{X} \in \tilde{\mathcal{D}}} \ell\left(\mathcal{X}_{i}, f_{\gamma}^{-1}\left(f_{\theta^{*}}\left(\mathcal{X}_{i}\right)\right)\right),
$$

where $\tilde{\gamma}$ is the estimated decoder from $\tilde{\mathcal{D}}$. Therefore we propose to apply a Gaussian noise to the computed signature to destroy the manifold structure, i.e., given a data entry $\mathcal{X}$, we compute the privacy-preserving signature $v$ by:

$$
v=f_{\theta^{*}}(\mathcal{X})+\epsilon, \quad \epsilon \sim \mathcal{N}\left(0, \sigma^{2}\right),
$$

where $\sigma^{2}$ is the variance controlling the magnitude of the noise, and should be chosen to balance the retrieval effectiveness and privacy level: a higher magnitude of noise gives stronger privacy protection but weakens the retrieval performance, and vice versa. We illustrate such trade-off in the empirical study section. The procedure of obtaining the signature is summarized in Figure 2.

\section{Similarity Retrieval}

Once a privacy-preserving signature $v$ is computed using (3), a data provider can then create a smart contract and publish the signature in the smart contract. Since the blockchain is publicly available, and data consumers can now get the list of signatures for all available data entries. In order to retrieve a set of relevant data entries to purchase, a consumer can complete the retrieval by comparing the signature of the purchase with a query signature $\hat{v}$. Directly comparing two signature vectors through cosine similarity $\cos (v, \hat{v})$ or inner product $v^{T} \hat{v}$ may not be effective, as the subspace of $v$ is chosen to maximize the reconstruction and thus may not be specific for the desired retrieval task. For example, one consumer wants to retrieval medical records that have prior diabetes diagnoses, and another consumer is interested in building models from those identified as dementia. For these two retrieval tasks, given the same query signature $\hat{v}$, the ranked list of data entities should look very different, because that the the definitions of "similarity" are different. From the machine learning perspective, the subspace learning for signature belongs to the unsupervised learning paradigm, and we need a supervised learning procedure and learn a similarity function $s_{t}(v, \hat{v})$ that is specific to task $t$, to carry out an efficient retrieval. Equivalently, we can learn a distance function $d_{t}(v, \hat{v})$ for the same purpose (by reversing the retrieved rank).

Let the $d_{t}$ be the Mahalanobis distance, i.e., $d_{t}(v, \hat{v})=$ $\sqrt{(v-\hat{v})^{T} M_{t}(v-\hat{v})}$, where $M_{t}$ is the parameter matrix that instructs how the distance should be computed for a specific task. Given the dataset $\mathcal{D}_{t}$ for retrieval task $t$, the parameter matrix $M_{t}$ can be estimated using a distance metric learning loss, e.g., the large-margin [22]. We denote the loss function as $\ell_{\varepsilon}$. When there are many retrieval tasks, their parameters can be estimated jointly using the multi-task learning paradigm, to transfer knowledge among tasks [22], [33]:

$$
\begin{aligned}
& \min _{M_{1}, \ldots, M_{t}} \sum_{t=1}^{T}\left(\sum_{(i, j) \in \mathcal{P}_{t}} d_{t}\left(v_{i}, v_{j}\right)^{2}+\right. \\
& \left.\sum_{(i, j, k) \in \mathcal{I}_{t}} \ell_{\varepsilon}\left(d_{t}\left(v_{i}, v_{j}\right), d_{t}\left(v_{j}, v_{k}\right)\right)\right), \\
& \text { s.t. } M_{t} \succeq 0, \forall t, \quad\left[M_{1}, \ldots, M_{T}\right] \in \mathcal{M},
\end{aligned}
$$

where $d_{t}$ is parameterized by $M_{t}, \mathcal{P}_{t}$ is the partial order set in dataset $\mathcal{D}_{t}$, and $\mathcal{I}_{t}$ is the triplet order set in $\mathcal{D}_{t}$ and each triple $(i, j, k)$ indicates the distance between $d_{t}\left(v_{i}, v_{j}\right)$ is closer than $d_{t}\left(v_{i}, v_{k}\right)$, and the positive semi- definiteness of $M_{t}$ makes the objective a convex programming. The subspace $\mathcal{M}$ constrains the solution spaces of $M_{t}$ and connects them to enable knowledge transfer. The subspace structure can be defined using many of existing multi-task learning approaches [33], such as shared subspace basis, sparsity patterns, or composite structures. 


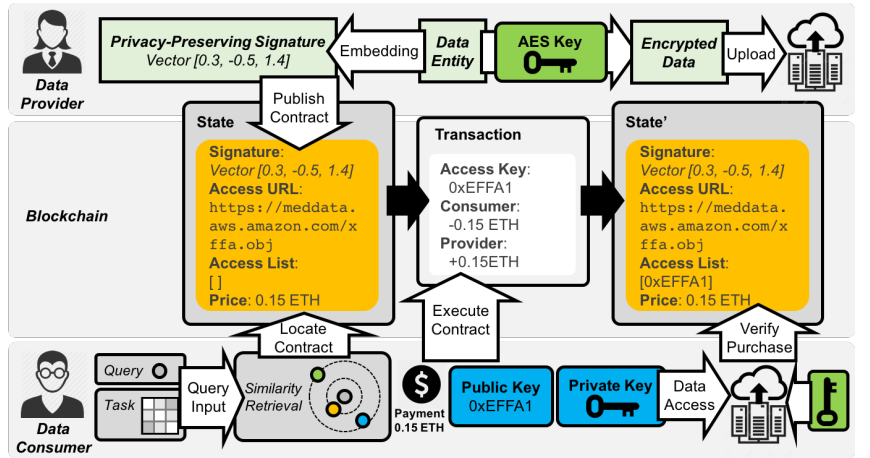

Fig. 3: Illustration of workflow for the proposed distributed data vending.

The multi-task metric learning approach can effectively improve the performance of distance metrics when there are limited amount of data supervision available, which improves the generalization of metrics through knowledge transfer. The simpler single task learning approach can be used once a large amount of training data is available, which reduces the additional bias introduced by multi-task learning priors. When distance metrics are learned for retrieval tasks, the metrics can be published online to a publicly available metric library, with the descriptions of tasks that they are associated to.

\section{Distributed Data Vending Workflow}

In this section we summarize the workflow of the proposed DDV framework. We assume the public availability of the following building blocks: the encoder $f_{\theta^{*}}$ and a metric library that includes a set of well-trained distance metrics. These metrics can be updated over time to improve the effectiveness of the retrieval.

a) Data Provider: A data provider is an entity who owns private data and can potentially sell the data pieces on blockchain.

b) Provider Device: A provider device is owned and trusted by a data provider. A typical provider device can be a smartphone. When a data provider wants to sell a piece of data, the raw data is firstly formatted into a data entity, representing the data into a tensor (or matrix/vector). The encoder $f_{\theta^{*}}$ is then used to embed the data into a privacypreserving signature vector. After that the provider device generates an AES key and uploads the data encrypted by this key to a data server. The data provider then creates a smart contract in the blockchain as described above.

A purchase from any consumer triggers a decryption process where the data provider sends the AES key to the data consumer via a secure channel after handshaking. The provider device then regenerates an AES key, encrypts the data using the new AES key again and sends the new copy of encrypted data to the data server for the next purchase.

c) Data Server: A data server hosts the encrypted data entities from data providers. Whenever a download request is initiated by a data consumer on a certain data entity, the server first retrieves its associated smart contract in blockchain. When the data consumer is authenticated by its public key in the smart contract, the encrypted data can be be downloaded by the data consumer. The data server then requests a new encrypted data for the next download request. This data ensures that each time a download is verified, a new AES key will be used for the next download. This way each encrypted copy of data entity will be valid for only once, so that the data will not be at risk if the data server gets compromised. See remarks below for how the AES key is deployed and retrieved.

d) Blockchain: The blockchain infrastructure can be any chain that enables smart contracts, such as Ethereum [31] or VeChain [28]. Whenever a data provider lists a data entity for sale, a smart contract is created that includes the data signature, an access URL of the data server (e.g., an AWS server) or an API address for retrieval, a list of public keys that granted data access, as well as the selling price for the data access. The smart contract can also include many more details such as demographic information of the patient if the data is EHR.

e) Data Consumer: A data consumer starts with a data collection task in mind, uses an existing data for query or construct the query, and applies the embedding function to compute the query signature. Based on the retrieval task, the provider then obtains the task-specific distance metric to rank the similarity of data entries available for purchase. Once identified one (or more) smart contract that includes data for purchase, the consumer then provides the public key and the demanded payment to the blockchain to execute the contract. Once the transaction is confirmed in the blockchain, the consumer then authenticates itself using its private key to download encrypted data from the data server. Meanwhile, the data consumer retrieves the AES key from provider device via the established secure channel between them. The download begins once the payment is verified by the server and the data is decrypted using the AES key.

The workflow is summarized in Figure 3 . In the next section we show a concrete example how the proposed DDV can be used for EHR data vending.

Remarks on Authentication and Data Security: The AES key is generated by and stored on the provider device, while the encrypted data entity is stored on the data server. The isolation of the key and the encrypted data prevents unauthorized decryption in case a data server is compromised. Secure channels between provider devices and data consumers are established by handshaking using public/private keys of both parties. The distribution of AES keys are done via the secure channels. The one-time AES key and encrypted data entity have further enhanced the data security of the framework.

\section{Case Study: Vending Healthcare Records}

The limited access to medical data has been a major bottleneck to many medical studies. With more data available, clinicians and data scientists can develop more accurate disease models and drug effects to reduce health costs and save lives. We believe that the proposed DDV framework can be used to relieve the the scarcity of data in medical research by encouraging patients to share self-reported medical history through blockchain, and the data contribution is acknowledged 


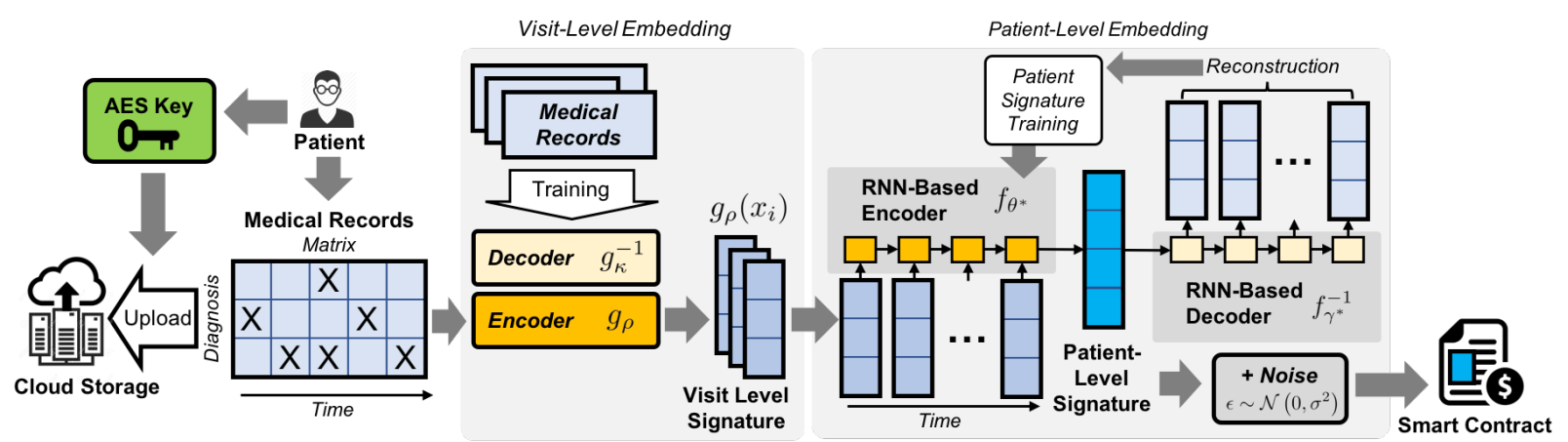

Fig. 4: Generating patient signature for vending medical records using the proposed DDV framework.

and rewarded by monetary incentives. In this section, we illustrate a prototype implementation of our DDV framework in the context of vending healthcare records. The implementation includes two components: (1) incrementally learns signature representations of longitudinal patient information and (2) produces task-specific similarity metric for data retrieval.

Although previous studies have cited the effectiveness of end-to-end sequence- to-sequence autoencoders over a wide array of representation tasks [17], substantial data is often required to obtain high-level performance using this approach. In the EHR setting, medical records often vary in sample size depending on the granularity of data available. For example, we have around 20 times more visit-level data than patientlevel temporal data in our experimental database. This means that a high-performing visit-level signature can be trained before sufficient data is available to drive high-performing patient-level signatures, which require many samples to capture both temporal patterns at the patient-level as well as sparsity patterns at the visit-level. Thus, we decompose the signature learning tasks into two sub-tasks, visit- and patientlevel signatures, so that our representation framework is better suited for incremental online learning.

a) Visit-Level Signatures: Obtaining the visit-level signature is a dimensional reduction task which takes visit vectors $x_{i} \in \mathcal{X}$ and outputs embedded vectors $g_{\rho}\left(x_{i}\right)$, where $g_{\rho}($. is the visit level signature function parameterized by $\rho$. In this case, we do not consider differences between patients and simply try to learn a low-dimensional representation of medical visits. For each visit, we consider all the relevant diagnostic ICD-9 group codes. Assume there are $d$ ICD-9 diagnosis group codes for consideration, and training inputs take on $x_{i} \in \mathbb{R}^{d}$ one-hot vector format, with 1's denoting group codes present for the visit. We then project the training vector into a $q$-dimensional dense latent space $g_{\rho}\left(x_{i}\right) \in \mathbb{R}^{q}$, and $q \ll d$.

We formulate the representation process as a multi-label classification problem, with $d$ binary classifiers trained in parallel for each visit sample. We learn the pair of encoder $g_{\rho}$ with the help of a decoder $g_{\kappa}^{-1}$ parameterized by $\kappa$. For a vector $x$, let $\hat{x}_{i}^{\rho, \kappa}=g_{\kappa}^{-1}\left(g_{\rho}\left(x_{i}\right)\right)$ be the reconstructed vector. The parameters $\rho, \kappa$ are then estimated from minimizing the following reconstruction error:

$$
\min _{\rho, \kappa} \sum_{j=1}^{d} \lambda_{j} \sum_{x_{i} \in \mathcal{X}}\left\{x_{i, j} \log \left(\hat{x}_{i, j}^{\rho, \kappa}\right)+\left(1-x_{i, j}\right) \log \left(1-\hat{x}_{i, j}^{\rho, \kappa}\right)\right\},
$$

where $\lambda_{j}$ is the weight that is given to the particular task $j \in[1, d]$ which correspond to the group code to be predicted during recovery. Depending on the sample, $\lambda_{j}$ assumes 1.0 if the sample is negative for the group code and 3.0 if the sample is positive for the group code during that visit. We used multi-layer perceptron to implement both $g_{\rho}$ and $g_{\kappa}^{-1}$.

b) Patient-Level Signatures: Once visit-level signature embedding is learned, the original EHR tensor $\mathcal{X}$ can be transformed into $\mathcal{G}$, which contains embedded visits for each patient across time. This way, when a vectorized representation of sequential patient information, we by-pass the sparse recovery problem mentioned in the previous section. Therefore, in (11) we simply minimize over the mean-squared error (MSE) between the original input $\mathcal{G}$ and the recovered tensor $f^{-1}(\mathcal{G})$ from the embedding process:

$$
\ell\left(G_{i}, f_{\gamma}^{-1}\left(f_{\theta}\left(\mathcal{G}_{i}\right)\right)\right)=\left\|\mathcal{G}_{i}-f_{\gamma}^{-1}\left(f_{\theta}\left(\mathcal{G}_{i}\right)\right)\right\|_{F}^{2},
$$

where $f_{\theta}$ and $f_{\gamma}^{-1}$ are two recurrent neural networks (RNN). Specifically, we applied long-short term memory (LSTM) units to construct RNNs for the encoder and decoder. We note that the number of visits differ for patients, so the number of visit vectors recovered per patient depends on the input patient. Thus, the RNN vector representation of $\mathcal{G}$ is robust to variations in visit frequency of patients. The entire procedure for generating patient signature is summarized in Figure 4.

\section{EXPERIMENTS}

In this section, we demonstrate the proposed distributed data vending (DDV) framework using medical records as elaborated in Section IV] We first introduce the dataset used for training the signatures and similarity functions, then assess the embedding performance and retrieval performance of DDV on the real dataset, and finally discuss how the different noise levels protect the signature and effect retrieval performance.

a) Dataset: We use a real EHR dataset from a hospital that includes more than 283,000 patients with documented diagnostic histories. A typical patient diagnostic history consists of a list of relevant ICD-9 diagnostic group codes for each visit as well as a summary ICD-9 problem list for the patient. We 
emphasize here that the difference between ICD-9 group codes and ICD-9 codes lies in the fact that group codes consist of 3letter representations of original ICD-9 codes. For example, a '250.00' is the ICD-9 code for type-II diabetes (T2DM) w/o specified complications, while ' 250 ' is the general ICD9 group code for type-II diabetic-related diseases, including T2DM with and without various specified complications. The group codes are typically used in feature representation of patients, while the ICD-9 codes themselves provide greater granularity in terms of querying during cohort-selection.

b) Embedding Performance of Patient-Level Signatures: In the representation task, we try to capture medical history information in the EHR database by finding a matrix representation $f_{\theta}\left(g_{\rho}(\mathcal{X})\right)=\mathbf{X}^{\prime}$ of the visit histories of each patient over the entire dataset tensor, $\mathcal{X}$. Here, the vector representation $\mathbf{x}^{\prime}{ }_{i} \in \mathbf{X}^{\prime}$ should ideally capture the sparsity features as well as the local temporal informations from the diagnostic history matrix $\mathbf{x}_{i} \in \mathcal{X}$ for each patient. We therefore evaluate the recovery performance of our representation across each visit and at each timestep for all patients.

In total, there are 1041 group codes in our dataset, so training inputs take on $\mathbf{x}_{i} \in \mathbb{R}^{1041}$ one-hot vector format, with 1's denoting group codes present for the visit. On average, each patient visit averages 3.69 group codes out of the possible 1041. Thus, we give greater weight to sparse, positive labels across all visits during training. Otherwise, an embedding which recovers all 0 entries over 1041 group codes for each visit can still achieve 0.99 accuracy due to the prominence of negative codes.

We evaluate the effectiveness of the patient-level autoencoder in two ways (1) given the de-noised signature vector for each patient, evaluate the micro-averaged precision and recall across all visits over 5 -fold validation and (2) after addition of Gaussian noise $\epsilon$ protection to each signature vector, re-train the decoder to recover the original $\mathcal{X}$ and evaluate performance over 5-fold validation. When a consumer purchases a substantial amount of medical records, the individual can theoretically train a decoder to recover the original medical dataset from the publicly available signature vectors. By adding Gaussian noise to the signatures, we can significantly limit the ability of an accurate decoder to be trained and generalized to the rest of the dataset. The noise-addition step thus serves as a privacy-preserving procedure over publicly available signature vectors. By comparing performance of the recovery processes in (1) and (2), we can gauge the upper limit in the level of granularity that a decoder is capable of recovering, given various levels of noise during the privacy-preserving process. Thus, (2) is included in our evaluation procedure as a measure of "decoder protection" which can be tracked in real time during deployment.

From Figure 5, we see that the embedding quality, as measured by recovery precision and recall over $\mathcal{X}$ from $f^{-1}\left(f_{\theta}\left(g_{\rho}((X))\right)\right)$, improves greatly with increasing intermediate signature vector representation size up to saturation point of around 2000 dimensions. At 6000 dimensions, signature vectors were able to capture over $90 \%$ of the sparse temporal

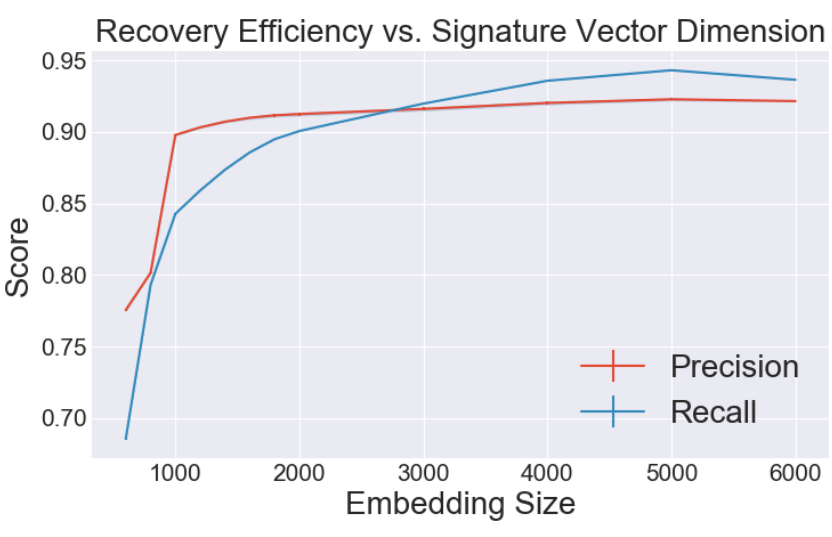

Fig. 5: Recovery efficiency of autoencoder framework using various signature vector dimension sizes.

features from the original data. This is unsurprising, as each visit vector from the original dataset was 1041 in dimension, and the average patient had 6.65 visits in their diagnostic histories. It is notable that at 2000 feature dimensions, recovery over $90 \%$ precision and recall was still achievable.

Figure 5 further illustrates the saturation of recovery efficiency with increase in signature vector sizes. Here we see that the shoulder of the performance curve occurs at 1000 dimensions, where increases in recovery efficiency decreases dramatically with additional embedding dimensions. For the purposes of scalable feature representation and retrieval performance, we want to use an embedding size which provides adequate compression of feature space from the original data without drastically sacrificing embedding accuracy. We thus recommend using 1000 dimensional embedding representation, as it provides over $7 \mathrm{x}$ compression of the original medical data while preserving majority of the sparsity and temporal properties for downstream tasks.

c) Decoder Protection: When considering embedding performance a key question to consider is: can we maintain the main information in the signature vector after adding protection noise? To answer this question we use the original decoder (jointly trained with the encoder) to recover data matrices from noise protected signature vectors. In Figure 6 we demonstrate the robustness of decoder recovery to privacypreserving Gaussian noise. Again, we witness an inflection point in the ability of the decoder to learn an accurate recovery of the original data under various noise levels. Here, we center the Gaussian noise at mean of $\mu=0.0$ and increase the standard deviation of noise, $\sigma=\epsilon$ where $\epsilon \in\{0.10,0.20,0.40,0.60,0.80,1.00,1.20,1.40,1.80,2.00\}$. At each noise level $\epsilon$, we construct a noise vector $\nu \in \mathbb{R}^{d}$, where $d$ is dimension of signature vector, and each component of $\nu$ consists of a random scalar drawn from $\mathcal{N}(\mu, \epsilon)$. For each vector, we construct a different noise vector, drawn from the same distribution under $\mathcal{N}(\mu, \epsilon)$. This noise vector then gets added to the original signature vector $\mathbf{X}_{i}$.

As seen in Table I] the steepest drop in recovery perfor- 
TABLE I: Recovery performance using 1000 dimensional signature under various noise levels.

\begin{tabular}{c||c|c}
\hline$\epsilon$ & Precision & Recall \\
\hline 0.10 & $0.841\left( \pm 8.8 \times 10^{-4}\right)$ & $0.953\left( \pm 3.1 \times 10^{-5}\right)$ \\
0.20 & $0.811\left( \pm 2.7 \times 10^{-5}\right)$ & $0.935\left( \pm 5.0 \times 10^{-6}\right)$ \\
0.40 & $0.511\left( \pm 2.9 \times 10^{-4}\right)$ & $0.882\left( \pm 2.0 \times 10^{-5}\right)$ \\
0.60 & $0.293\left( \pm 2.8 \times 10^{-4}\right)$ & $0.791\left( \pm 5.1 \times 10^{-5}\right)$ \\
0.80 & $0.202\left( \pm 5.3 \times 10^{-5}\right)$ & $0.672\left( \pm 1.8 \times 10^{-5}\right)$ \\
1.00 & $0.160\left( \pm 6.6 \times 10^{-6}\right)$ & $0.553\left( \pm 2.8 \times 10^{-5}\right)$ \\
1.20 & $0.124\left( \pm 2.3 \times 10^{-5}\right)$ & $0.464\left( \pm 7.5 \times 10^{-5}\right)$ \\
1.40 & $0.108\left( \pm 6.8 \times 10^{-6}\right)$ & $0.400\left( \pm 7.7 \times 10^{-5}\right)$ \\
1.80 & $0.084\left( \pm 9.3 \times 10^{-7}\right)$ & $0.300\left( \pm 2.2 \times 10^{-6}\right)$ \\
2.00 & $0.080\left( \pm 1.3 \times 10^{-6}\right)$ & $0.268\left( \pm 2.5 \times 10^{-6}\right)$ \\
\hline
\end{tabular}

mance occurred between $0.40 \leq \epsilon \leq 0.60$, where precision decreased from above $80 \%$ to below $50 \%$. Further increases in noise levels facilitate the exponential decrease in decoder recovery, yet it is interesting to note that the exponential decay of decoder performance did not occur until noise level reached a substantial level. This is most likely due to the inherent robustness of the decoder to noise at the input level. Another interesting pattern to note is that added noise induced more false-positive predictions by the decoder, which is indicated by the increasing between precision and recall scores with increasing noise levels.

Given the retrieval performance under noise protection, one key question still remains: can we prevent users from recovering the decoder when they have accumulated a reasonable amount of protected signatures? We answer this question by measuring decoder parameter changes during the re-training procedure by first vectorizing the weight layers in the decoders and then taking the Euclidean distance between the de-noised parameters $\theta_{0}$ and re-trained parameters $\theta_{\epsilon}$ at each noise level. Table II, examines these parametric differences between the original decoder and the recovered one. One clear trend is that as the magnitude of noise increases, differences in parameter decrease during re-training. With increasing noise, the decoder is less capable of learning correct parameters due to the increasing randomness in the added noise. It is important to note that while increase in noise level can provide privacy over the signature vector signatures, the optimal level of noise must be fine tuned against retrieval efficiency. In the following section, we demonstrate the trade-off between the protectiveness of high $\epsilon$ noise-levels and the usability of the transformed signature vectors for retrieval.

d) Retrieval Performance: From the standpoint of the consumer, a typical query order on a medical database include selecting for patients with specific ICD-9 codes for downstream cohort studies. For this reason, we formulate the retrieval task as searching for top $N$ patients, given a specific cohort. We constructed 10 mutually exclusive cohorts, distinguished by ICD-9 codes. Table III summarizes the number of available patients for each cohort. In total, there were 58,489 patients in this subset of the EHR database. Each patient has a unique label in one-hot vector format, $\mathbf{y} \in \mathbb{R}^{10}$. The retrieval task is formulated as follows: given a cohort of interest, $\mathbf{y}$,

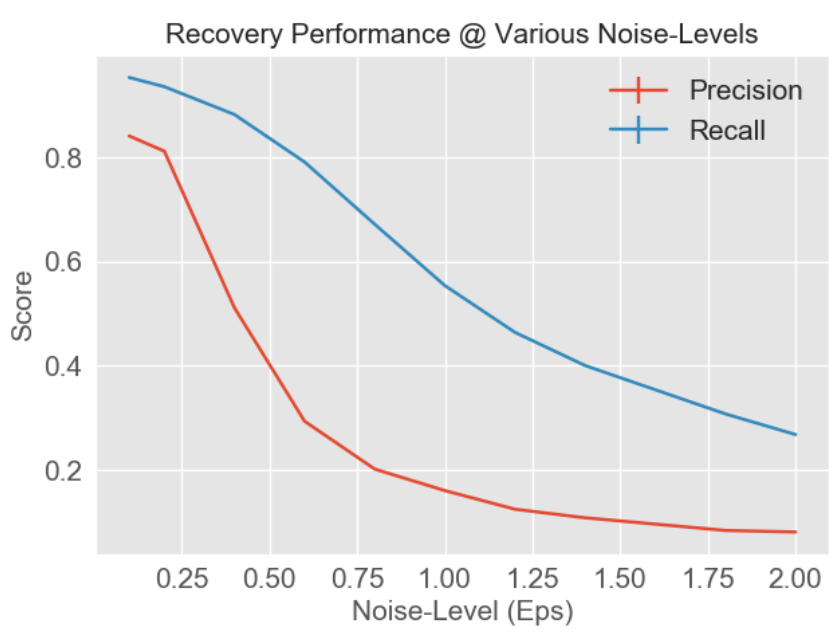

Fig. 6: Decoder recovery performance at various noise levels on 1000 dimensional vector signatures.

TABLE II: Changes in learned decoder parameters with increasing noise.

\begin{tabular}{c||c|c}
\hline$\epsilon$ & $\left\|\theta_{\epsilon}-\theta_{0}\right\|_{2}^{2}$ & $\left\|\theta_{\epsilon}-\theta_{0}\right\|_{2}^{2} /\left\|\theta_{0}\right\|_{2}^{2}$ \\
\hline 0.10 & 91.96 & 0.402 \\
0.20 & 90.56 & 0.395 \\
0.40 & 85.94 & 0.376 \\
0.60 & 85.75 & 0.375 \\
0.80 & 87.51 & 0.382 \\
1.00 & 90.16 & 0.393 \\
1.20 & 83.70 & 0.366 \\
1.40 & 81.64 & 0.357 \\
1.80 & 81.53 & 0.355 \\
2.00 & 81.64 & 0.360 \\
\hline
\end{tabular}

find the top $N$ patients that belonging to this cohort.

As mentioned in the previous sections, a similarity matrix $M_{t}$ is trained using multi-task metric learning [22] for each task $\mathbf{y}_{t} \in\left\{\mathbf{y}_{1}, \ldots, \mathbf{y}_{10}\right\}$. Then, a characteristic query vector $\mathbf{q}_{t}$ is obtained for each task by taking the component-wise average of signature vectors across the cohort. For example, the congestive heart failure (CHF) cohort contains 1, 456 unique patients, each represented by a signature vector $\mathbf{x}_{i}^{\prime} \in \mathbb{R}^{d}$. The cohort can thus be represented by a $1,456 \times d$ matrix, $\mathbf{X}_{\text {CHF }}^{\prime}$. To perform the retrieval task, we take the characteristic query vector to be $\mathbf{q}=\frac{1}{m} \sum_{i}^{m} \mathbf{x}_{i}^{\prime}$, where $m=1,456$. Once this query vector is obtained, $M_{t}$ can be used to calculate the distance for each patient in the cohort, where $d_{t}\left(\mathbf{q}_{t}, \mathbf{x}_{i}^{\prime}\right)=\sqrt{\left(\mathbf{q}_{t}-\mathbf{x}_{i}^{\prime}\right) M_{t}\left(\mathbf{q}_{t}-\mathbf{x}_{i}^{\prime}\right)}$. We then rank the top $N$ "closest" patients to the target query by Mahalanobis distance and use them for retrieval evaluation.

We evaluate the performance of each retrieval task by calculating the precision@ $N$ score for $N \in\{10,50,100,250,500\}$. By comparing precision at various retrieval sizes $(N)$, we can deduce the scalability of various queries, given the metrics $M_{t} \in \mathcal{M}$ learned by [22]. Table $\mathrm{V}$ demonstrates these differences in scalability, as we can see that for tasks such as MCI, CAD and CHF, retrieval precision decreases dramatically 
TABLE III: Summary of cohorts for retrieval tasks.

\begin{tabular}{c|c|c|c}
\hline ICD-9 Code & Description & Raw Count & Exclusive \\
\hline \hline 250.00 & Type-II DM & 39,699 & 13,381 \\
427.31 & Arrythmias & 21,072 & 4,734 \\
311 & Depression & 29,978 & 9,863 \\
272.4 & Hyperlipidemia & 48,302 & 12,500 \\
414.00 & CAD & 22,936 & 3,249 \\
244.9 & Hypothyroidism & 22,416 & 6,167 \\
162.9 & Lung Cancer & 5,397 & 2,927 \\
428.0 & CHF & 13,516 & 1,456 \\
174.9 & Breast Cancer & 8,379 & 3,672 \\
331.83 & MCI & 2,473 & 540 \\
\hline Total & \multicolumn{3}{|c}{58,489} \\
\hline \multicolumn{4}{|c}{}
\end{tabular}

TABLE IV: Multi-task LMNN classification error rates.

\begin{tabular}{l|c|c}
\hline Task & Validation & Testing \\
\hline \hline T2DM & 2.750 & 2.621 \\
Arrythmia & 4.912 & 6.604 \\
Depression & 2.292 & 3.564 \\
Hyperlipidemia & 6.549 & 6.918 \\
CAD & 3.274 & 4.298 \\
Hypothyroidism & 3.602 & 3.826 \\
Lung Cancer & 0.655 & 0.786 \\
CHF & 3.274 & 3.878 \\
Breast Cancer & 1.834 & 1.992 \\
MCI & 1.965 & 2.306 \\
\hline
\end{tabular}

after 100 cohorts, while for tasks such as T2DM, lung cancer and breast cancer retrieval, precision remains near $100 \%$ even at much larger retrieval sizes. By contrast, the actual largest margin nearest neighbors (MT-LMNN) performance, as shown in Table [V] indicates that the classification error remain consistently below $7 \%$ across all tasks when classification is done in a pair-wise manner between candidate patients. The cross-cohort discrepancy between query-based retrieval and MT-LMNN performance highlights the importance of query design for accurate retrieval under our framework.

For example, in the case of T2DM, predictable patterns of comorbidity often ensue the progression of the disease. Endstage renal diseases (ESRD), ophthalmic and neurosensory complications inevitably co-occur with T2DM during the longitudinal development of symptomatology. On the other hand, CHF does not follow a singular, predictable pattern of development. In fact, CHF itself can be decomposed into several subtypes of progression, all of which result from vastly different compensatory pathways. Since the retrieval task relies on a single query representation $\mathbf{q}_{t}$ per cohort, techniques such as signature-vector averaging and singular vector selection may not adequately capture the variability within certain cohorts. Future studies may focus on designing effective ways to produce query vector representations which can cover such variability within disease phenotypes.

e) Effect of Noise on Retrieval: As mentioned previously, privacy-preserving noise produces a trade-off in performance between recovery and retrieval: addition of high noise may decrease decoder recovery, thereby increasing privacy, yet it may also decrease retrieval performance. We examine the
TABLE V: Retrieval performance of learned cohort-specific metrics using 1000 dimensional de-noised signatures. Top performing queries include Type-II Diabetes Mellitus (T2DM), lung and breast cancers, all of which achieved $\geq 99 \%$ precision at various retrieval sizes.

\begin{tabular}{c|c|c|c|c|c}
\hline Cohort & Prc@ 10 & Prc@50 & Prc@ 100 & Prc@250 & Prc@ 500 \\
\hline \hline T2DM & 1.000 & 1.000 & 1.000 & 1.000 & 1.000 \\
Arrythmia & 1.000 & 1.000 & 1.000 & 0.684 & 0.408 \\
Breast Cancer & 1.000 & 1.000 & 1.000 & 0.996 & 0.988 \\
CAD & 1.000 & 0.935 & 0.581 & 0.352 & 0.194 \\
CHF & 1.000 & 0.940 & 0.466 & 0.200 & 0.106 \\
Depression & 1.000 & 1.000 & 1.000 & 1.000 & 0.686 \\
Hyperlipidemia & 1.000 & 1.000 & 0.794 & 0.784 & 0.608 \\
Hypothyroid & 1.000 & 0.981 & 0.979 & 0.624 & 0.408 \\
Lung Cancer & 1.000 & 1.000 & 1.000 & 1.000 & 1.000 \\
MCI & 1.000 & 0.781 & 0.443 & 0.184 & 0.100 \\
\hline
\end{tabular}

effect of noise on retrieval performance by re-learning similarity matrices $M_{t} \in \mathcal{M}$ for each individual task while using the increasingly noisy versions of signature vectors. Retrieval performance was again evaluated using precision@ $N$ at each noise level $\epsilon \in\{0.0,0.2,0.4,0.6,0.8,1.0\}$ across various retrieval tasks. This time, we included only the top performing retrieval tasks, including: T2DM, lung cancer, breast cancer, depression and arrhythmia. These tasks possess the highest performing queries from the de-noised retrieval setting.

As shown in Figures $7 \mathrm{~b}-7 \mathrm{f}$, precision scores drop dramatically with increasing noise levels. With $\epsilon>0.6$, the recovery behavior becomes more erratic and may not follow the monotonically decreasing trend as seen with the typical case. Figures $7 \mathrm{~b}$ and $7 \mathrm{c}$ demonstrate the noise-levels that are capable of producing accurate retrievals for majority of the tasks. When comparing with the privacy-preserving performance shown in Figure 6, we see that the optimal noise level for 1000 dimension signatures is $\epsilon=0.4$, as this level of noise disrupts decoder learning enough to limit the consumer's capacity to recover the original dataset while maintaining precision during retrieval. We notice that in both recovery and retrieval, noise levels beyond $\epsilon=0.6$ leads to erratic behavior. In the case of recovery, decoder performance decreases exponentially with increasing noise above 0.6 , but retrieval performance suffers greatly in response.

\section{CONCLUSION}

In this paper, we studied the problem of distributed data vending, which allows personal data to be securely exchanged on a blockchain. We proposed a framework for distributed data vending through a combination of data embedding and similarity learning, to tackle the dilemma between the effectiveness of data retrieval, and the leakage from indexing the data. Distributed data vending can transform domains such as healthcare by encouraging data distribution from owners and its aggregation. We illustrated our framework through a practical scenario of distributing electronic medical records on a blockchain. We conducted extensive empirical results to demonstrate the effectiveness of our framework. 


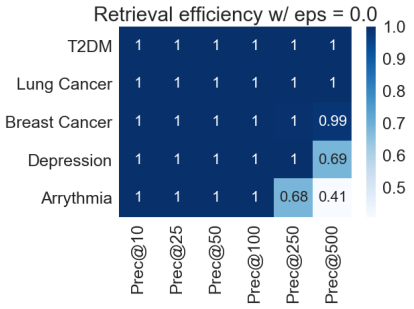

(a) $\epsilon=0.0$

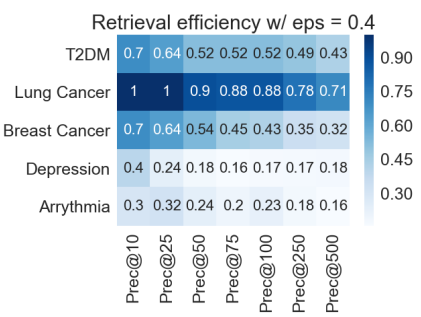

(c) $\epsilon=0.4$

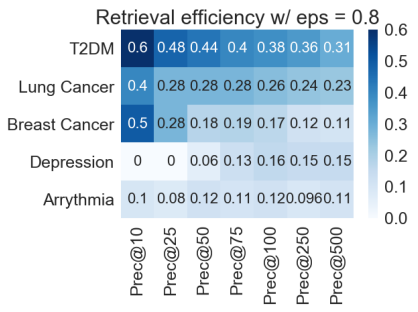

(e) $\epsilon=0.8$

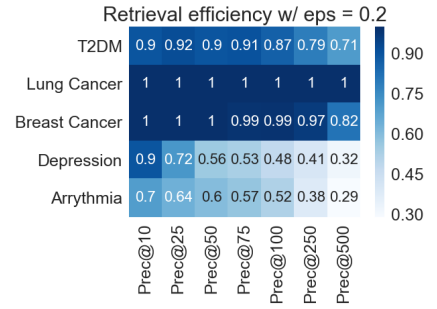

(b) $\epsilon=0.2$

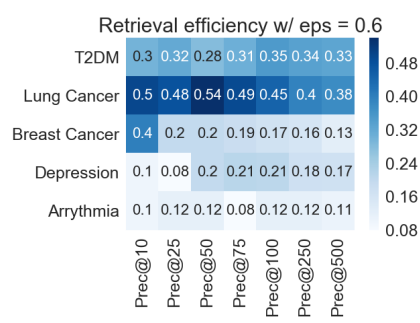

(d) $\epsilon=0.6$

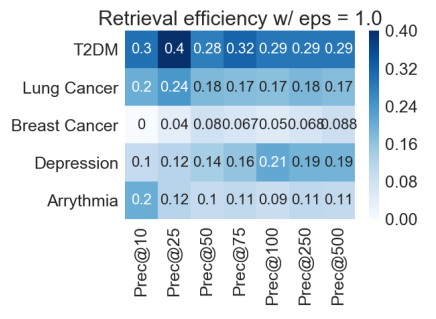

(f) $\epsilon=1.0$
Fig. 7: Heatmap displays of retrieval efficiency across various retrieval demands with increasing noise levels.

\section{ACKNOWLEDGMENT}

This research is supported in part by VeChain Foundation. We thank Chi Jin from Michigan State for discussions during the work.

\section{REFERENCES}

[1] A. Azaria, A. Ekblaw, T. Vieira, and A. Lippman. Medrec: Using blockchain for medical data access and permission management. In Open and Big Data (OBD), International Conference on, pages 25-30. IEEE, 2016.

[2] G. Baxendale. Can blockchain revolutionise eprs? ITNow, 58(1):38-39, 2016.

[3] C. Brodersen, B. Kalis, C. Leong, E. Mitchell, E. Pupo, and A. Truscott. Applying blockchain technology to medicine traceability, 2016.

[4] S.-A. Brown. Patient similarity: Emerging concepts in systems and precision medicine. Frontiers in physiology, 7:561, 2016.

[5] Y. Cui, N. Shivakumar, A. Carobus, D. Jindal, and S. Lawrence. Contenttargeted advertising using collected user behavior data, Jan. 27 2005. US Patent App. 10/649,585.

[6] A. Ekblaw and A. Azaria. Medrec: Medical data management on the blockchain. Ariel, 1(10):7, 2016.

[7] L. Esposito. How to get access to your hospital records, 2014.

[8] J. A. Greene and W. V. Padula. Targeting unconscionable prescriptiondrug pricesmarylands anti-price-gouging law. New England Journal of Medicine, 377(2):101-103, 2017.

[9] M. Iansiti and K. R. Lakhani. The truth about blockchain. Harvard Business Review, 95(1):118-127, 2017.

[10] I. T. Jolliffe. Principal component analysis and factor analysis. In Principal component analysis, pages 115-128. Springer, 1986.
[11] A. Karatzoglou, X. Amatriain, L. Baltrunas, and N. Oliver. Multiverse recommendation: $\mathrm{n}$-dimensional tensor factorization for context-aware collaborative filtering. In Proceedings of the fourth ACM conference on Recommender systems, pages 79-86. ACM, 2010.

[12] Y. Koren, R. Bell, and C. Volinsky. Matrix factorization techniques for recommender systems. Computer, 42(8), 2009.

[13] T.-T. Kuo, H.-E. Kim, and L. Ohno-Machado. Blockchain distributed ledger technologies for biomedical and health care applications. Journal of the American Medical Informatics Association, 24(6):1211-1220, 2017.

[14] T.-T. Kuo and L. Ohno-Machado. Modelchain: Decentralized privacy-preserving healthcare predictive modeling framework on private blockchain networks. arXiv preprint arXiv:1802.01746, 2018.

[15] G. Linden, B. Smith, and J. York. Amazon. com recommendations: Item-to-item collaborative filtering. IEEE Internet computing, 7(1):7680, 2003.

[16] S. Livingston. Health insurers' proposed 2018 rate hikes are early 'warning signs', 2018.

[17] M.-T. Luong, Q. V. Le, I. Sutskever, O. Vinyals, and L. Kaiser. Multitask sequence to sequence learning. arXiv preprint arXiv:1511.06114, 2015.

[18] M. Mettler. Blockchain technology in healthcare: The revolution starts here. In e-Health Networking, Applications and Services (Healthcom), 2016 IEEE 18th International Conference on, pages 1-3. IEEE, 2016.

[19] S. Mika, G. Ratsch, J. Weston, B. Scholkopf, and K.-R. Mullers. Fisher discriminant analysis with kernels. In Neural networks for signal processing IX, 1999. Proceedings of the 1999 IEEE signal processing society workshop., pages 41-48. Ieee, 1999.

[20] S. Nakamoto. Bitcoin: A peer-to-peer electronic cash system. 2008.

[21] U. D. of Health, H. Services, et al. Summary of the hipaa privacy rule. Washington, DC: Author. Retrieved December, 2:2007, 2003.

[22] S. Parameswaran and K. Q. Weinberger. Large margin multi-task metric learning. In Advances in neural information processing systems, pages 1867-1875, 2010.

[23] K. Peterson, R. Deeduvanu, P. Kanjamala, and K. Boles. A blockchainbased approach to health information exchange networks. In Proc. NIST Workshop Blockchain Healthcare, volume 1, pages 1-10, 2016.

[24] I. Sutskever, O. Vinyals, and Q. V. Le. Sequence to sequence learning with neural networks. In Advances in neural information processing systems, pages 3104-3112, 2014.

[25] P. Taylor. Applying blockchain technology to medicine traceability, 2016.

[26] E. J. Topol. Money back guarantees for non-reproducible results?, 2016.

[27] F. Tschorsch and B. Scheuermann. Bitcoin and beyond: A technical survey on decentralized digital currencies. IEEE Communications Surveys \& Tutorials, 18(3):2084-2123, 2016.

[28] Vechain. Vechain, 2018.

[29] S. Velimeneti. Data migration from legacy systems to modern database. 2016.

[30] P. Vincent, H. Larochelle, Y. Bengio, and P.-A. Manzagol. Extracting and composing robust features with denoising autoencoders. In Proceedings of the 25th international conference on Machine learning, pages 10961103. ACM, 2008.

[31] G. Wood. Ethereum: A secure decentralised generalised transaction ledger. Ethereum Project Yellow Paper, 151:1-32, 2014.

[32] L. Yang and R. Jin. Distance metric learning: A comprehensive survey. Michigan State Universiy, 2(2), 2006.

[33] J. Zhou, J. Chen, and J. Ye. Malsar: Multi-task learning via structural regularization. Arizona State University, 2011.

[34] J. Zhou, J. Liu, V. A. Narayan, J. Ye, A. D. N. Initiative, et al. Modeling disease progression via multi-task learning. NeuroImage, 78:233-248, 2013.

[35] J. Zhou, Z. Lu, J. Sun, L. Yuan, F. Wang, and J. Ye. Feafiner: biomarker identification from medical data through feature generalization and selection. In Proceedings of the 19th ACM SIGKDD international conference on Knowledge discovery and data mining, pages 1034-1042. ACM, 2013.

[36] J. Zhou, F. Wang, J. Hu, and J. Ye. From micro to macro: data driven phenotyping by densification of longitudinal electronic medical records. In Proceedings of the 20th ACM SIGKDD international conference on Knowledge discovery and data mining, pages 135-144. ACM, 2014.

[37] H. Zhu, C. Huang, and J. Zhou. Edgechain: Blockchain-based multi-vendor mobile edge application placement. arXiv preprint arXiv:1801.04035, 2018. 